\title{
TOMOGRAPHIC APPROACH FOR SAMPLING MULTIDIMENSIONAL SIGNALS WITH FINITE RATE OF INNOVATION
}

\author{
$\dagger \ddagger$ Pancham Shukla and $\dagger$ Pier Luigi Dragotti \\ $\dagger$ Communications and Signal Processing Group, Electrical and Electronic Engineering, \\ Imperial College London, Exhibition Road, London SW7 2AZ, England. \\ † G H Patel College of Engineering \& Technology, Vallabh Vidyanagar 388120, Gujarat, India. \\ Email: \{P.Shukla, P.Dragotti\}@imperial.ac.uk
}

\begin{abstract}
Recently, it was shown that it is possible to sample classes of 1$\mathrm{D}$ and 2-D signals with finite rate of innovation (FRI) [9, 4, 5, 3, $2,7]$. In particular, in [7], we presented local and global schemes for sampling sets of Diracs and bilevel polygons using compactly supported kernels that reproduce polynomials.

In sequel to [7], in this paper, we present a Radon transform based hybrid scheme for sampling more general FRI signals such as piecewise polynomials with polygonal boundaries, and higher dimensional Diracs and bilevel polytopes. The key feature of the proposed scheme is an annihilating-filter-based-back-projection (AFBP) algorithm.
\end{abstract}

Index Terms - Signal sampling, Signal representations, Signal resolution, Signal reconstruction, Radon transforms, Polynomial approximation, Singular value decomposition, Spectral analysis, Wavelet transforms, Nonbandlimited signals.

\section{INTRODUCTION}

Sampling plays an important role in modern signal processing and communication applications. Shannon's classical sampling theory and its extensions are very powerful and have been successfully utilized for bandlimited signals. Moreover, classical sampling is also extended for classes of non-bandlimited signals that reside in a shiftinvariant subspace [8].

Recently, novel sampling schemes $[9,4,5]$ are presented for larger classes of 1-D and 2-D signals that are neither bandlimited nor reside in a fixed subspace. Such signals have a finite number of degrees of freedom (or rate of innovation) and are classified as signals with Finite Rate of Innovation (FRI) [9]. The sampling schemes of $[9,4,5]$ rely on annihilating filter method and Fourier domain proceeding. Moreover, these schemes employ infinite support sinc and Gaussian kernels, and for that reason, are not convenient in practice.

However, in $[3,7,2]$, it was shown that many 1-D and 2-D FRI signals with local rate of innovation can be sampled and perfectly reconstructed using compactly supported kernels that satisfy StrangFix conditions, and therefore, reproduce polynomials. In this paper, we extend the results of [7] for sampling more general 2-D FRI signals such as piecewise polynomials with polygonal boundaries, and higher dimensional Diracs and polytopes. We employ an annihilating filter based back projection (AFBP) algorithm that utilizes a link between Radon transform projections and moments [6].

The research was supported by the Engineering and Physical Sciences Research Council (EPSRC) of UK under the grant GR/S57631/01.
The paper is organized as follows: In the next section, we develop an AFBP algorithm by integrating the Radon-moment connection [6] in the framework of FRI sampling [5, 3, 2]. In Section 3, we derive the sampling results for bilevel polygons, 2-D Diracs, and piecewise polynomials with polygonal boundaries using AFBP algorithm. We then show that the sampling of 2-D Diracs and bilevel polygons can be extended to higher dimensions (i.e. in 3-D and above). Finally, we conclude in Section 4.

\section{TOMOGRAPHIC APPROACH}

\subsection{Radon transform}

Let $g(x, y)$ be a 2-D square-integrable function within a compact region $\Omega$ over the Euclidean space $\mathbb{R}^{2}$. Then, the conventional Radon transform projection of $g(x, y)$ is defined as (also see Figure 1(a)) [1]:

$R_{g}(t, \theta)=\iint_{\Omega} g(x, y) \delta(t-x \cos (\theta)-y \sin (\theta)) d x d y$,

where $l_{t, \theta}=\delta(t-x \cos (\theta)-y \sin (\theta))$ is a straight line of integration at an angle $\theta+\frac{\pi}{2}$ with the $x$-axis and at a radial distance $t$ away from the origin. From Figure 1(a), note that the projection gathering line $l_{t, \theta}^{\perp}$ that makes an angle $\theta \in[0, \pi)$ with the $x$-axis is perpendicular to $l_{t, \theta}$. The projections $R_{g}(t, \theta)$ are square integrable 1-D functions with finite support. The original function $g(x, y)$ can again be reconstructed from its projections $R_{g}(t, \theta)$ using filtered back-projection (FBP) reconstruction [1].

\subsection{AFBP algorithm}

For a specific case, when $g(x, y)$ is a bilevel and convex polygon with $N$ corner points, we observe that

(a) Each projection $R_{g}(t, \theta)$ is a 1-D piecewise polynomial of maximum degree one and with at most $N$ discontinuities. Therefore, the 2 nd order derivative of such projection leads to a stream of at most $N$ differentiated Diracs $d_{t}^{(2)}\left[R_{g}(t, \theta)\right]=$ $\frac{d^{2}}{d t^{2}}\left[R_{g}(t, \theta)\right]=\sum_{i=0}^{N-1} \sum_{r=0}^{1} a_{i, r} \delta^{(r)}\left(t-t_{i}\right)$, where $t_{i}$ are locations and $a_{i, r}$ are weights. It means that $d_{t}^{(2)}\left[R_{g}(t, \theta)\right]$ represents at most $N$ Diracs with $\hat{N}=2 N$ weights [3,2].

(b) Moreover, following the connection between Radon projections and moments [6], the moments $\mu_{n}$ of the differentiated Diracs 


$$
\begin{aligned}
d_{t}^{(2)} & {\left[R_{g}(t, \theta)\right] \text { are obtained by } } \\
\mu_{n} & =\int d_{t}^{(2)}\left[R_{g}(t, \theta)\right] t^{n} d t \\
& =\iint_{\Omega} d_{t}^{(2)}[g(x, y)](x \cos (\theta)+y \sin (\theta))^{n} d x d y \\
& =\sum_{\beta=0}^{n}\left(\begin{array}{l}
n \\
\beta
\end{array}\right) \cos ^{\alpha}(\theta) \sin ^{\beta}(\theta) \mu_{\alpha, \beta},
\end{aligned}
$$

where $\mu_{\alpha, \beta}$ are $n=(\alpha+\beta)$-order geometric moments of the differentiated polygon $d_{t}^{(2)}[g(x, y)]=\frac{d^{2}}{d t^{2}}[g(x, y)]$.

(c) Since $d_{t}^{(2)}\left[R_{g}(t, \theta)\right]$ consists of at most $N$ Diracs with $\hat{N}=2 N$ weights, the $2 \hat{N}=4 N$ moments $\mu_{n}, n=0,1, \ldots, 4 N-1$ are sufficient to retrieve the locations $t_{i}$ and weights $a_{i, r}$ of the Diracs $d_{t}^{(2)}\left[R_{g}(t, \theta)\right]$ (and therefore the piecewise polynomial signal $R_{g}(t, \theta)$ itself) using annihilating filter method [3, 2].

(d) By iterating the steps (a), (b), and (c) over $N+1$ distinct projection angles $\theta_{l}, l=0,1, \ldots, N$, the $N+1$ sets of Dirac locations $t_{i}$ are determined. By back-projecting these locations, the $N$ corner points of $g(x, y)$ are retrieved. Since the bilevel polygon $g(x, y)$ is convex, the corner points can uniquely recover $g(x, y)$ [5].

Since the retrieval of corner points is based on annihilating filter, we denote the proposed reconstruction as: annihilating filter based backprojection (AFBP) algorithm. Note that the AFBP algorithm can be extended to retrieve the corner points of the piecewise polynomials with polygonal boundaries and higher dimensional polytopes.

\subsection{Example: AFBP reconstruction of a bilevel polygon}

As shown in Figure 1, consider a bilevel and convex polygon $g(x, y)$ with $N=5$ corner points . From Figure 1(b), it is clear that the projection $R_{g}(t, \theta)$ along $\theta=0$ is a 1-D piecewise polynomial of degree one with $N$ discontinuities, and therefore by taking two successive derivatives, it can be decomposed into a stream of $\mathrm{N}$ 1-D Diracs as depicted in Figure 1(d). That is $d_{t}^{(2)}\left[R_{g}(t, \theta)\right]=$ $\frac{d^{2}}{d t^{2}}\left[R_{g}(t, \theta)\right]=\sum_{i=0}^{N-1} \sum_{r=0}^{1} a_{i, r} \delta^{(r)}\left(t-t_{i}\right)$, where locations $t_{i}$ of the Diracs determine the back-projection points $c_{i}^{b p}$, and the coefficients $a_{i, r}$ are the weights.

Fortunately, in this case $(\theta=0)$, we have $N$ Diracs with $\hat{N}=N$ weights since all $a_{i, 1}=0$. Therefore, from step (c) of the AFBP algorithm, we know that the first $2 \hat{N}=2 N$ moments $\mu_{n}$ are sufficient to determine the exact locations $t_{i}$ of the $N$ Diracs $d_{t}^{(2)}\left[R_{g}(t, \theta)\right]$. To be more precise, using (2), we obtain the $2 N$ moments $\mu_{n}, n=0,1, \ldots, 2 N-1$ and design an annihilating filter $A[n], n=0,1, \ldots, N$ such that the convolution

$$
\mu_{n} * A[n]=0 \text {. }
$$

Consequently, the $N$ roots of the filter $A[n]$ give the exact locations $t_{i}$ of the stream of $N$ Diracs $d_{t}^{(2)}\left[R_{g}(t, \theta)\right]$. Therefore, the coordinates of the corresponding back-projection points $c_{i}^{b p}, i=$ $1,2, \ldots, N$ along the projection gathering line $l_{t, \theta}^{\perp}$ are given by $\left(x_{i}^{b p}, y_{i}^{b p}\right)=\left(\left|t_{i}\right| \cos (\theta),\left|t_{i}\right| \sin (\theta)\right)$. In this case $(\theta=0)$, the back-projection points $c_{i}^{b p}$ are marked with $\mathbf{\square}$ on the $x$-axis in Figure 1(a).

In a similar manner, we obtain $N+1$ distinct projections $d_{t}^{(2)}\left[R_{g}\left(t, \theta_{l}\right)\right]$ along $\theta_{l}, l=0,1, \ldots, N$. Then, using (2) and (3),

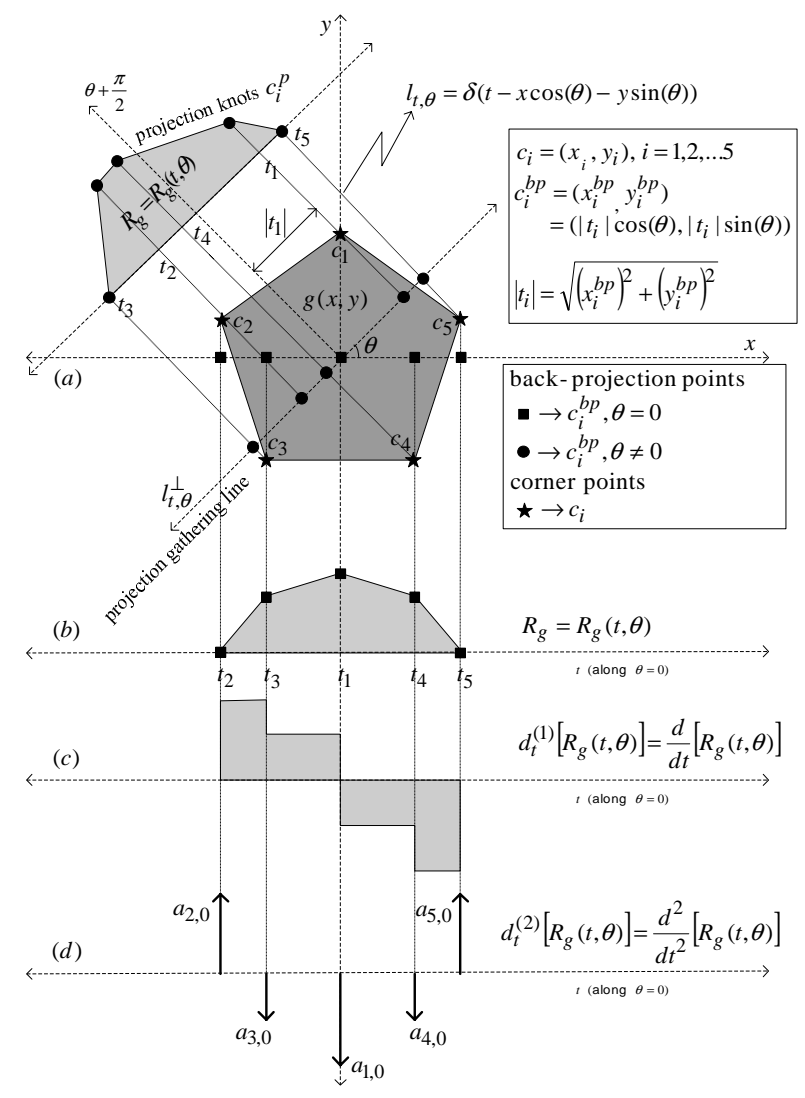

Fig. 1. Radon transform projection and AFBP reconstruction: For a bilevel pentagon $g(x, y)$, its Radon transform projection $R_{g}(t, \theta)$ along an arbitrary angle $\theta$ is shown in part (a). The projection of $g(x, y)$ along $x$-axis $(\theta=0)$ is shown in (b). The projection in part (b) is a 1-D piecewise linear function, and therefore, can be represented by a stream of Diracs as shown in part (d).

we retrieve $N+1$ sets of Dirac locations $t_{i}$, and therefore $N+1$ sets of back-projection points $c_{i}^{b p}$ along respective projection gathering lines $l_{t, \theta_{l}}^{\perp}$. By projecting back these $N+1$ sets of back-projection points, we retrieve $N$ corner points $c_{i}$ of the polygon $g(x, y)$, and thus the polygon itself. Note that, in general, $4 N$ moments $\mu_{n}$ of the $N+1$ differentiated Radon projections $d_{t}^{(2)}\left[R_{g}\left(t, \theta_{l}\right)\right]$ are sufficient to reconstruct the convex and bilevel polygon $g(x, y)$ with $N$ corner points.

Equipped with the Radon-moment connection (2) and AFBP algorithm, we now present a sampling perspective to the tomographic reconstruction of FRI signals. ${ }^{1}$ For clarity, we concentrate on bilevel polygons.

\section{SAMPLING OF FRI SIGNALS}

\subsection{Sampling setup and kernel}

Consider a generic sampling setup in 2-D, where a continuous FRI signal $g(x, y)$ is prefiltered with a smoothing kernel $\varphi_{x y}(x, y)$, and

\footnotetext{
${ }^{1}$ The signals, we consider, include sets of 2-D Diracs, bilevel polygons, piecewise polynomials with polygonal boundaries, and higher dimensional Diracs and polytopes.
} 
the filtered version $g(x, y) * \varphi_{x y}\left(-x / T_{x},-y / T_{y}\right)$ is sampled uniformly to obtain a set of samples $S_{j, k}$ given by

$$
S_{j, k}=\left\langle g(x, y), \varphi_{x y}\left(x / T_{x}-j, y / T_{y}-k\right)\right\rangle,
$$

where $T_{x}, T_{y} \in \mathbb{R}^{+}$are the sampling intervals along $x$ and $y$ directions respectively, and for simplicity, assume $T_{x}=T_{y}=1$.

The sampling kernel $\varphi_{x y}(x, y)$ is of compact support, and can reproduce polynomials up to degree $n=\alpha+\beta$. That is, there exists coefficients $C_{j, k}^{\alpha, \beta}$ such that the kernel $\varphi_{x y}(x, y)$ satisfies:

$$
\sum_{j=-\infty}^{\infty} \sum_{k=-\infty}^{\infty} C_{j, k}^{\alpha, \beta} \varphi_{x y}(x-j, y-k)=x^{\alpha} y^{\beta},
$$

where $\alpha, \beta$ specify the degrees of polynomials that the sampling kernel $\varphi_{x y}(x, y)$ can reproduce along $x$ and $y$ respectively. For example, the scaling functions (from wavelet theory) and B-spines are the kernels that reproduce polynomials.

The polynomial reproduction property of the sampling kernel allows us to obtain the moments $\mu_{\alpha, \beta}$ of the original signal $g(x, y)$ from its samples $S_{j, k}$ as given by

$$
\mu_{\alpha, \beta}=\iint_{\Omega} g(x, y) x^{\alpha} y^{\beta} d x d y=\sum_{j} \sum_{k} S_{j, k} C_{j, k}^{\alpha, \beta} .
$$

This result is, in fact, at the heart of our sampling schemes.

\subsection{Bilevel polygons}

Assume that we have access to a convex and bilevel polygon $g(x, y)$ with $N$ corner points in form of its samples $S_{j, k}$ given by (4) using a kernel $\varphi_{x y}(x, y)$ that can reproduce polynomials up to degree $4 N-$ 3 . Recall that in order to retrieve the corner points of $g(x, y)$, we need to compute the moments $\mu_{n}$ of the differentiated projections $d_{t}^{(2)}\left[R_{g}(t, \theta)\right]$ from the moments $\mu_{\alpha, \beta}$ of the differentiated polygon $d_{t}^{(2)}[g(x, y)]$ as given in (2).

Nevertheless, from lattice theory, it is possible to show that there exists a direction vector $\vec{v}=\left[v_{x}, v_{y}\right]$ along a chosen projection angle $\theta=\tan ^{-1}\left(\frac{v_{y}}{v_{x}}\right), v_{x}, v_{y} \in \mathbb{Z}$ such that the discrete domain directional differences $\mathcal{D}_{\theta}^{(2)}\left[S_{j, k}\right]$ and continuous domain directional derivatives $d_{t}^{(2)}[g(x, y)]$ follow [7]

$$
\begin{aligned}
R_{j, k}= & \mathcal{D}_{\theta}^{(2)}\left[S_{j, k}\right] \\
= & {\left[S_{\left(j+2 v_{x}\right),\left(k+2 v_{y}\right)}-S_{\left(j+v_{x}\right),\left(k+v_{y}\right)}\right]-} \\
& {\left[S_{\left(j+v_{x}\right),\left(k+v_{y}\right)}-S_{j, k}\right] } \\
= & \left\langle d_{t}^{(2)}[g(x, y)], \zeta_{\theta}(x-j, y-k)\right\rangle,
\end{aligned}
$$

where $R_{j, k}=\mathcal{D}_{\theta}^{(2)}\left[S_{j, k}\right]$, a new set of samples obtained by the second order directional difference on the set of samples $S_{j, k}$, is equivalent to the differentiated polygon $d_{t}^{(2)}[g(x, y)]$.

The directional kernel $\zeta_{\theta}(x, y)$ is produced by two successive convolutions of the original sampling kernel $\varphi_{x y}(x, y)$ with the zero-th order 1-D B-spline $\beta_{\theta}^{0}(x, y)$ in the direction of $\vec{v}$. More precisely, $\zeta_{\theta}(x, y)=|v|^{2}\left(\varphi_{x y}(x, y) * \beta_{\theta}^{0}(x, y)\right) * \beta_{\theta}^{0}(x, y)$.

It is important to note that the directional kernel also satisfies the polynomial reproduction property of (5). In particular, if the sampling kernel $\varphi_{x y}(x, y)$ can reproduce polynomials up to degree $4 N-3$, then the directional kernel $\zeta_{\theta}(x, y)$ can reproduce polynomials up to degree $4 N-1$ along $\theta$. The polynomial reproduction property of the directional kernel $\zeta_{\theta}(x, y)$ allows us to obtain the moments $\mu_{\alpha, \beta}$ of the differentiated polygon $d_{t}^{(2)}[g(x, y)]$ from its samples $R_{j, k}=\mathcal{D}_{\theta}^{(2)}\left[S_{j, k}\right]$ using

$\mu_{\alpha, \beta}=\iint_{\Omega} d_{t}^{(2)}[g(x, y)] x^{\alpha} y^{\beta} d x d y=\sum_{j} \sum_{k} R_{j, k} C_{j, k}^{\alpha, \beta}$.

Consequently, by importing (8) in equation (2), the moments $\mu_{n}$ of the differentiated Diracs $d_{t}^{(2)}\left[R_{g}(t, \theta)\right]$ are given by

$$
\mu_{n}=\sum_{\beta=0}^{n}\left(\begin{array}{l}
n \\
\beta
\end{array}\right) \cos ^{\alpha}(\theta) \sin ^{\beta}(\theta)\left(\sum_{j} \sum_{k} R_{j, k} C_{j, k}^{\alpha, \beta}\right),
$$

where $C_{j, k}^{\alpha, \beta}$ are the coefficients associated with the kernel $\zeta_{\theta}(x, y)$, and $n=\alpha+\beta$.

In fact, we obtain first $4 N$ moments $\mu_{n}, n=0,1, \ldots, 4 N-1$ of the differentiated projections $d_{t}^{(2)}\left[R_{g}\left(t, \theta_{l}\right)\right]$ from the linear combinations of samples $R_{j, k}$ and coefficients $C_{j, k}^{\alpha, \beta}$ along $N+1$ projection angles $\theta_{l}, l=0,1, \ldots, N$. Then following the steps (c) and (d) of the AFBP algorithm, we retrieve $N$ corner points of the polygon $g(x, y)$ and thus the polygon itself. In summary we have,

Proposition 1. Given a convex and bilevel polygon $g(x, y)$ with $N$ corner points, a set of samples $S_{j, k}=$ $\left\langle g(x, y), \varphi_{x y}\left(x / T_{x}-j, y / T_{y}-k\right)\right\rangle$ is sufficient to determine the first $4 N$ moments $\mu_{n}$ of the Radon transform projections $d_{t}^{(2)}\left[R_{g}\left(t, \theta_{l}\right)\right], l=0,1, \ldots, N$ along $N+1$ distinct angles $\theta_{l}$ with $\tan \left(\theta_{l}\right) \in \mathbb{Q}$ and thus the polygon $g(x, y)$ itself, provided that the sampling kernel $\varphi_{x y}(x, y)$ can reproduce polynomials up to degree $4 N-3$.

Algorithm: Given a valid set of samples $S_{j, k}$, the AFBP reconstruction of the polygon $g(x, y)$ with $N$ corner points follows the following steps:

1. For a chosen angle $\theta=\tan ^{-1}\left(\frac{v_{y}}{v_{x}}\right), v_{x}, v_{y} \in \mathbb{Z}$, compute the difference $R_{j, k}=\mathcal{D}_{\theta}^{(2)}\left[S_{j, k}\right]$ given by (7).

2. Using (9), compute the first $4 N$ moments $\mu_{n}, n=$ $0,1, \ldots, 4 N-1$ of the projection $d_{t}^{(2)}\left[R_{g}(t, \theta)\right]$ from the new set of samples $R_{j, k}$.

3. From moments $\mu_{n}$, using (3), obtain the exact locations $t_{i}, i=1,2, \ldots, N$ of $N$ Diracs $d_{t}^{(2)}\left[R_{g}(t, \theta)\right]$ and thus the back-projection points $c_{i}^{b p}=\left(\left|t_{i}\right| \cos (\theta),\left|t_{i}\right| \sin (\theta)\right)$.

4. Iterate steps 1,2 , and 3 for $N+1$ distinct projection angles $\theta_{l}, l=0,1, \ldots, N$.

5. By back-projecting $N+1$ sets of back-projection points $c_{i}^{b p}$, retrieve $N$ corner points $c_{i}$ of the polygon $g(x, y)$, and thus the polygon itself.

For simplicity, simulation result for a bilevel triangle $g(x, y)$ is shown in Figure 2. The original polygon $g(x, y)$ and the reconstructed corner points (marked with + ) are shown in part (a). The samples $S_{j, k}=\left\langle g(x, y), \varphi_{x y}\left(x / T_{x}-j, y / T_{y}-k\right)\right\rangle$ are shown in part (b), where $\varphi_{x y}(x, y)$ is a B-spline sampling kernel that can reproduce polynomials up to degree $4 N-3=9$ along both $x$ and $y$ directions, and therefore, the associated directional kernel $\zeta_{\theta}(x, y)$ can reproduce polynomials up to degree $4 N-1=11$ along $\theta$. The sets of differentiated samples $R_{j, k}=D_{\theta_{l}}^{2}\left[S_{j, k}\right], l=0,1, \ldots, N$ along four distinct angles $\theta_{0}=0, \theta_{1}=\frac{\pi}{4}, \theta_{2}=\tan ^{-1}(2)$ and $\theta_{3}=\frac{\pi}{2}$ are shown in parts (c), (d), (e), and (f). The AFBP reconstruction of the corner points $c_{i}, i=1,2, \ldots, N$ (marked with $\circ$ ) using $N+1=4$ sets of back-projections is shown in part $(\mathrm{g})$. 


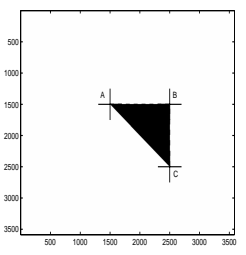

(a)

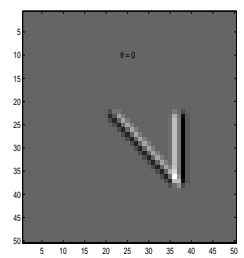

(c)

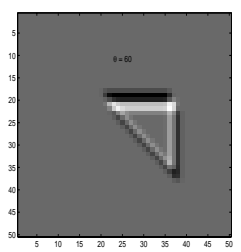

(e)

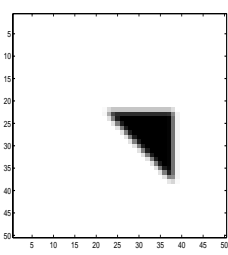

(b)

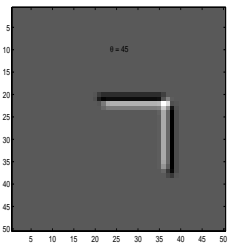

(d)

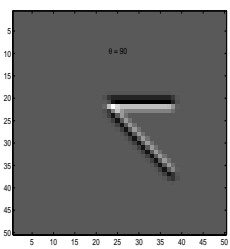

(f)

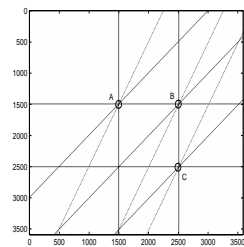

$(\mathrm{g})$

Fig. 2. Simulation: The original bilevel triangle $g(x, y)$, and the reconstructed corner points $A, B$, and $C$ (marked with + ) are given in part (a). The set of samples $S_{j, k}$ produced by the B-spline sampling kernel $\beta_{x y}^{9}$ is given in part (b). The $N+1=4$ sets of differentiated samples $R_{j, k}=D_{\theta}^{(2)}\left[S_{j, k}\right]$ along four angles $\theta=0, \frac{\pi}{4}, \tan ^{-1}(2)$, and $\frac{\pi}{2}$ are given in parts (c), (d), (e), and (f). The AFBP reconstruction of the corner points $A, B$, and $C$ is illustrated in part (g).

\subsection{Diracs and piecewise polynomials}

If $g(x, y)$ is a set of $N$ 2-D Diracs then each projection $R_{g}\left(t, \theta_{l}\right), l=$ $0,1, \ldots, N$ is a stream of at most $N$ Diracs with $\hat{N}=N$ weights. Clearly, in this case, Proposition 1 can be extended using a kernel that can reproduce polynomials up to degree $2 N-1$. Moreover, there is a freedom in selecting any $N+1$ distinct angles $\theta_{l}$ in the interval $[0, \pi)$, since there is no need to differentiate the projections $R_{g}\left(t, \theta_{l}\right)$.

Now assume that $g(x, y)$ is a piecewise polynomial of degree $R-1$ inside a convex polygonal closure $\Omega$ with $N$ corner points. In this case: 1) The convex closure $\Omega$ is recovered by employing the AFBP algorithm. 2) Once the closure $\Omega$ is known, the polynomial inside the polygon is determined from a finite number of Radon projections $R_{g}\left(t, \theta_{l}\right)$ by solving a system of linear equations. Note that, for this case Proposition 1 can be extended using a kernel $\varphi_{x y}(x, y)$ that can reproduce polynomials up to degree $(2 N-1)(R+1)-1$.

\subsection{Multidimensional extension}

The Radon transform $R_{g}(u, \vec{\lambda})$ of a square-integrable $n$-D function $g(\vec{x})$ with $\vec{x}=\left(x_{1}, x_{2}, \ldots, x_{n}\right)$ is defined as [1]

$$
R_{g}(u, \vec{\lambda})=\int_{\mathbb{R}^{n}} g(\vec{x}) \delta(\vec{\lambda} \vec{x}-u) d \vec{x},
$$

where $\|\vec{\lambda}\|=1$ and $\vec{\lambda} \vec{x}=u$ is an $(n-1)$-dimensional manifold orthogonal to $\vec{\lambda}$. Usually, Radon transform of a higher-dimensional $(n \geq 3)$ object is hierarchically decomposed into its 2 -D equivalents. The back-projection reconstruction is achieved via bottom-up approach.

It is possible to show that $n$-dimensional sets of $N$ Diracs or $n$-D bilevel and convex polytopes with $N$ corner points can be reconstructed exactly from a finite number of 1-D projections. For instance, it is straightforward to realize that $N$ 3-D Diracs can be reconstructed by using $N+12$-D projections, and therefore, by using $(N+1)^{2}$ projections in 1-D. In general, a set of $N$ Diracs (or a bilevel and convex polytope with $N$ corner points) in $n$-D can be reconstructed by using $(N+1)^{n-1} 1$-D projections. The proof of this assertion is an $n$-dimensional extension to its equivalent in 2-D [5].

From a sampling point of view, we can obtain the higher order moments of $(N+1)^{n-1} 1$-D projections of the $n$-D signals (i.e. $N$ Diracs or bilevel polytopes with $N$ corner points) from their samples, and then using AFBP algorithm, we can reconstruct these signals exactly.

\section{CONCLUSION}

In this paper, we have extended our sampling results [7] for more general FRI signals using a tomographic approach. We presented AFBP reconstruction of the original FRI signal $g(x, y)$ from its samples rather than sampling its Radon transform projections $R_{g}(t, \theta)$ [5].

\section{REFERENCES}

[1] S. R. Deans "The Radon Transform and Some of Its Applications," John Wiley, 1983.

[2] P. L. Dragotti, M. Vetterli, and T. Blu "Sampling moments and reconstructing signals with finite rate of innovation: Shannon meets Strang-Fix," IEEE Trans. on Signal Processing, February 2006, submitted.

[3] P. L. Dragotti, M. Vetterli, and T. Blu, "Exact sampling results for signals with finite rate of innovation using Strang-Fix conditions and local kernels," Proc. IEEE ICASSP, Philadelphia, USA, March 2005.

[4] I. Maravic and M. Vetterli, "Exact sampling results for some classes of parametric nonbandlimited 2-D signals," IEEE Trans. on Signal Processing, 52(1):175-189, January 2004.

[5] I. Maravic and M. Vetterli, "A sampling theorem for the Radon transform of finite complexity objects," Proc. IEEE ICASSP, Orlando, Florida, May 2002.

[6] P. Milanfar, G. Verghese, W. Karl, and A. Willsky, "Reconstructing polygons from moments with connections to array processing," IEEE Trans. on Signal Processing, 43:432-443, February 1995.

[7] P. Shukla and P. L. Dragotti, "Sampling schemes for 2-D signals with finite rate of innovation using kernels that reproduce polynomials," Proc. IEEE ICIP, Genova, Italy, September 2005.

[8] M. Unser, "Sampling- 50 Years after Shannon," Proc. IEEE, 88(4):569-587, April 2000.

[9] M. Vetterli, P. Marziliano, and T. Blu, "Sampling signals with finite rate of innovation," IEEE Trans. on Signal Processing, 50(6):1417-1428. June 2002. 